\title{
Effects of Mitochondrial Toxins on the Brain Amino Acid Concentrations
}

\author{
Peter Klivenyi, ${ }^{1}$ Katalin A. Kekesi, ${ }^{2}$ Zsuzsanna Hartai, ${ }^{1}$ Gabor Juhasz, ${ }^{3}$ \\ and Laszlo Vecsei ${ }^{1,4,5}$
}

\begin{abstract}
In the pathogenesis of Parkinson's disease and Huntington's disease excitotoxicity may play an important role. The common toxin model for Parkinson's disease is MPTP, while for Huntington's disease it is 3-NP. These toxins inhibit the mitochondrial respiratory chain, resulting in an energy deficit. In the central nervous system, the amino acids act as neurotransmitters and neuromodulators. The energy deficit caused by these neurotoxins may alter the concentrations of amino acids. Thus, it can be claimed that the aminoacidergic neurotransmission can be changed by neurotoxins. To test this hypothesis we studied the amino acid concentrations in different brain regions following MPTP or 3-NP administration. The two toxins were found to produce similar changes. We detected marked decreases in most of the amino acid concentrations in the striatum and in the cortex, while the levels in the cerebellum increased significantly. The decreased amino acid levels can be explained by the reduced levels of ATP produced by these neurotoxins. In the cerebellum, where there is no detectable ATP loss, the elevated amino acid levels may reflect a compensation of the altered neurotransmission.
\end{abstract}

KEY WORDS: Amino acids; amino acid transport; cerebellum; compensation; cortex; energy deficit; excitotoxicity; Huntington's disease; MPTP; neurotransmission; 3 nitropropionic acid; Parkinson's disease; striatum.

\section{INTRODUCTION}

The mechanism of nerve cell death in neurodegenerative diseases is currently extensively studied. The view of a central role for excitotoxicity and

${ }^{1}$ Department of Neurology, University of Szeged, Szeged, Hungary.

${ }^{2}$ Department of Neurobiology and Physiology, Eötvös Loránd University, Budapest, Hungary.

${ }^{3}$ Research Group of Neurobiology, Hungarian Academy of Sciences and Eötvös Loránd University, Budapest, Hungary.

${ }^{4}$ Neurology Research Group, Hungarian Academy of Science and University of Szeged, Szeged, Hungary.

${ }^{5}$ Address reprint requests to: Laszlo Vecsei, Department of Neurology, University of Szeged, P.O.B. 427, H-6701, Szeged, Hungary; Fax: +36-62-545597; E-mail: vecsei@nepsy.szote. u-szeged.hu oxidative damage in the pathogenesis of Parkinson's disease (PD) and Huntington's disease (HD) is gaining increasing acceptance (1-3). There is also substantial evidence linking mitochondrial dysfunction and free radical formation (4).

A commonly used neurotoxin model for PD is 1-methyl-4-phenyl-1,2,5,6-tetrahydropyridine (MPTP). Following systemic administration to both human and nonhuman primates, MPTP results in biochemical and neuropathological features, which closely resemble PD. MPTP is metabolized by monoamine oxidase B (MAO B) to 1-methyl-4-phenylpyridinium $\left(\mathrm{MPP}^{+}\right)$, which is taken up into the dopaminergic neurons by the synaptic dopamine transporter. $\mathrm{MPP}^{+}$accumulates in the mitochondria, where it inhibits complex I of the electron transport chain 
$(5,6)$. This may subsequently lead to indirect excitotoxicity and free radical generation (7). Inhibitors of MAO B completely prevent the MPTP neurotoxicity.

The neurotoxin 3-nitropropionic acid (3-NP) has been well characterized as an experimental model of HD (8). This toxin inhibits succinate dehydrogenase, inducing a mitochondrial dysfunction which triggers the generation of superoxide radicals and secondary excitotoxicity leading to a selective striatal pathology closely resembling to the pathology found in patients with HD (6).

Amino acids are important neurotransmitters and neuromodulators in the central nervous system. Above a certain concentration, glutamate (Glu), aspartate (Asp) and glycine (Gly) exert neurotoxic effects via an excitotoxic mechanism, while the functions of other amino acids are not fully known. Amino acids may be transported via neutral amino acid transporters, which require ATP (9). Blockade of the mitochondrial respiratory chain by mitochondrial toxins results in an energy deficit, which may alter the amino acid levels. These changes can enhance the neurotoxic effect. The respiratory chain inhibition performs an endproduct inhibition to the citric acid cycle, which is the major source of organic acid precursors of amino acids. Particularly $\alpha$-ketoglutarate is involved in metabolism of glutamate. Only limited data are available regarding the effects of these toxins on the amino acid levels. Decreased Glu and Asp levels have been found in the striatum and mesencephalon, but not in the cerebellar cortex, which recovered after $24 \mathrm{~h}(10)$.

In the present study, we investigated the effects of MPTP and 3-NP on the levels of amino acids. We hypothesized that changes in the amino acid levels combined with increased free radical generation caused by mitochondrial toxins may contribute to the irreversible cell loss. Specifically, we sought to establish whether this mechanism affects all brain parts equally.

\section{MATERIALS AND METHODS}

All animal experiments were carried out in accordance with the European Union Guide for the Care and Use of Laboratory Animals and were approved by the local animal care committee.

MPTP (Sigma, St. Louis, USA) was dissolved in phosphatebuffered saline (PBS) and a single dose $(30 \mathrm{mg} / \mathrm{kg}$ ) was injected ip in a volume of $0.15 \mathrm{ml}$ into CFLP male mice (weight $30 \mathrm{~g}$ ). The control mice received PBS vehicle.
3-NP (Sigma, St. Louis, USA) was dissolved in PBS $(\mathrm{pH}$ adjusted to 7.4) and injected intraperitoneally at a dose of $50 \mathrm{mg} /$ $\mathrm{kg}$ into the same mouse strain. Seven animals were examined in each group and seven mice served as control group for both toxins. Mice were sacrificed 3, 6, 12 or $24 \mathrm{~h}$ after MPTP or 3-NP injection. The brains were quickly removed and the entire striata, the frontal cortex and the cerebellum were dissected on an ice-cold plate and kept at $-70^{\circ} \mathrm{C}$ until measurements were made. For the dissection we used mouse brain matrices for slicing the exact intervals.

Sample Preparation for Amino Acid Analysis. A 1-5 mg piece of each brain sample was placed into an Eppendorf tube containing $20 \mu$ leluent B. The wet tissue weight was measured with $0.01 \mathrm{mg}$ accuracy. Tissue samples were homogenized with a Teflon potter homogenizer for $10 \mathrm{~s}$. The potter speed was $6000 \mathrm{rpm}$. The homogenized samples were treated with a $1000 \mathrm{~W}$ microwave beam for $10 \mathrm{~s}$. The samples were then centrifuged at $12,000 \mathrm{rpm}$ for $20 \mathrm{~min}$ in an Eppendorf centrifuge. About $10 \mu \mathrm{l}$ supernatants were stored for no more than 1 week at $-20^{\circ} \mathrm{C}$; they were then analyzed by HPLC.

Concentrations of amino acids were measured by precolumn derivatization of primary amino acids with orthophthalaldehyde (OPA). Derivatization was performed at $\mathrm{pH} 10.4$ in the presence of mercaptoethanol. OPA-derivatized amino acids were detected with fluorescence detector excitation wavelength $340 \mathrm{~nm}$ and emission wavelength $440 \mathrm{~nm}$. Because of the instability of OPA derivatives, the HPLC technique was automated on an HP 1100 series system. Amino acids were separated on HP Hypersyl ODS reversed-phase columns $(200 \times 2.1 \mathrm{~mm})$ filled with $5 \mu \mathrm{m}$ $\mathrm{C}-18$ spherical packing material. Eluent $\mathrm{A}$ was $0.1 \mathrm{M}$ phosphate buffer containing $0.5 \%(\mathrm{vol} / \mathrm{vol})$ tetrahydrofuran, $\mathrm{pH} 6.0$, while eluent B contained $70 \%$ acetonitrile in eluent $\mathrm{A}, \mathrm{pH}$ 6.0. The gradient profile was: $12 \% \mathrm{~B}$ at $0 \mathrm{~min}, 23 \% \mathrm{~B}$ at $9 \mathrm{~min}, 36 \% \mathrm{~B}$ at $18 \mathrm{~min}, 100 \% \mathrm{~B}$ at $19 \mathrm{~min}, 100 \% \mathrm{~B}$ at $22 \mathrm{~min}$ and $12 \% \mathrm{~B}$ at $26 \mathrm{~min}$. The column equilibration time was $8 \mathrm{~min}$. External standards of $10 \mu \mathrm{M}$ amino acids were injected after every 20 samples (11). Chromatograms were evaluated with HP ChemStation software.

One-way analysis of variance followed by the Fisher protected least significance difference test was used to analyze the data. A $p$ value of less than 0.05 was considered statistically significant.

\section{RESULTS}

\section{After MPTP Administration}

The levels of the amino acids in different brain regions of mice treated with MPTP are shown in Figs. 1, 2 and 3.

In the striatum, 3 and $6 \mathrm{~h}$ after MPTP injection, the concentrations Asp, Asn, Ser, Gly, Arg, Ala and GABA were decreased, but the levels recovered almost completely after $24 \mathrm{~h}$. The concentrations of Glu, Gln, Thr and Tau did not change significantly.

After MPTP administration, the levels of Asp, Asn, Ser, Gly, Thr, Arg, Ala, Tau and GABA decreased in the cortex, and remained low for $24 \mathrm{~h}$. Interestingly, the levels of Gln and Glu were decreased significantly only after 12 and $24 \mathrm{~h}$. 

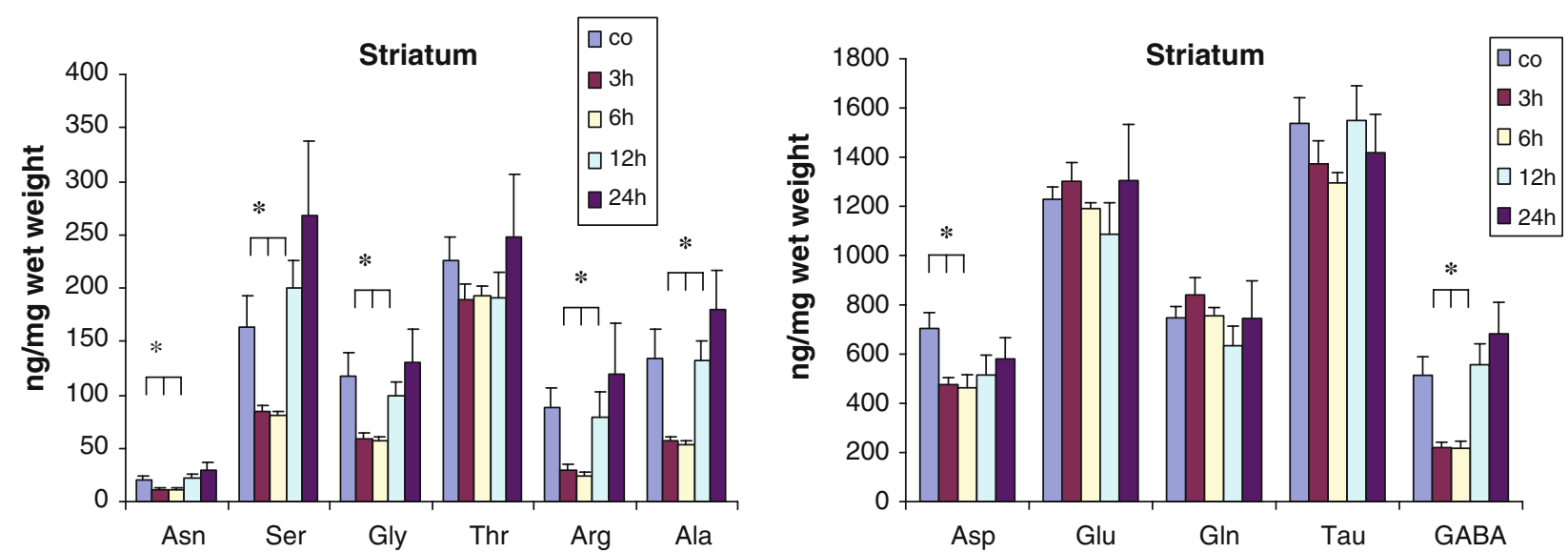

Fig. 1. Concentration of amino acids in the striatum after MPTP treatment $(\mathrm{n}=7$ mice/groups). $* 3$ and $6 \mathrm{~h}$ levels are significantly decreased compared with the controls $(P<0.05 ; F(4,40)=2.5 ; 3.0 ; 2.5 ; 0.5 ; 2.0 ; 2.3 ; 2.3 ; 0.69 ; 1.0 ; 4.0$ respectively).

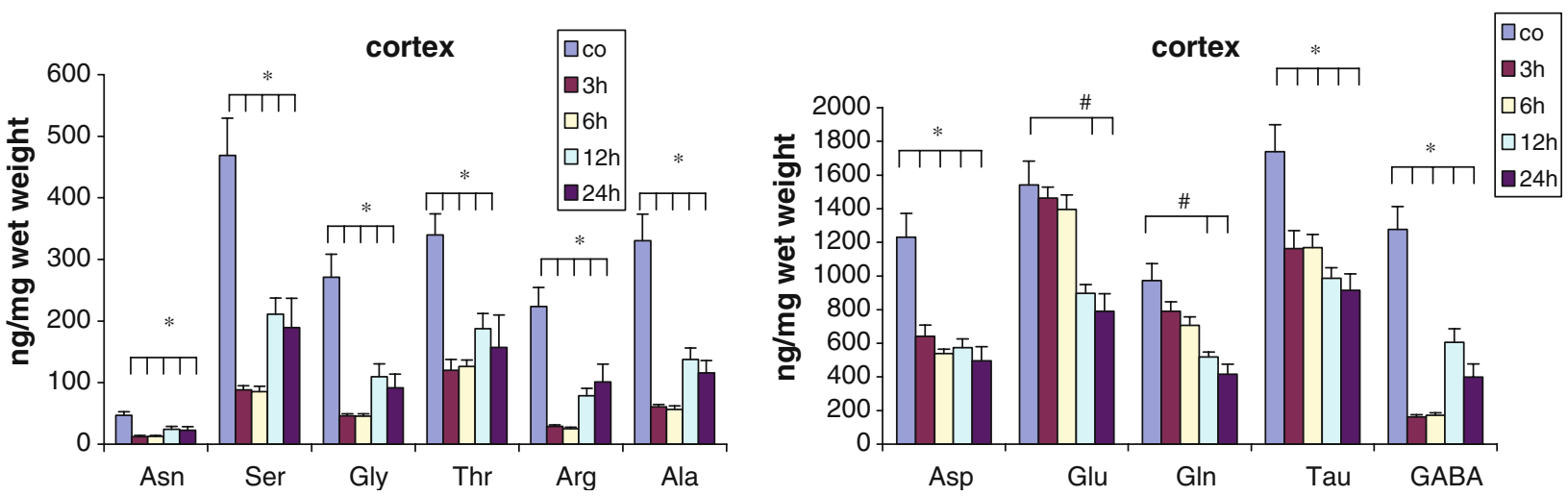

Fig. 2. Concentration of amino acids in the cortex after MPTP treatment ( $\mathrm{n}=7$ mice/groups). *at all time-points the levels are significantly decreased compared with the controls $(P<0.05)$. ${ }^{\#} 12$ and $24 \mathrm{~h}$ levels are significantly decreased compared with the controls $(P<0.05$; $F(4,33)=9.6 ; 11.7 ; 11.3 ; 8.2 ; 11.0 ; 13.3 ; 8.6 ; 7.6 ; 7.4 ; 7.1 ; 20.4$ respectively $)$.
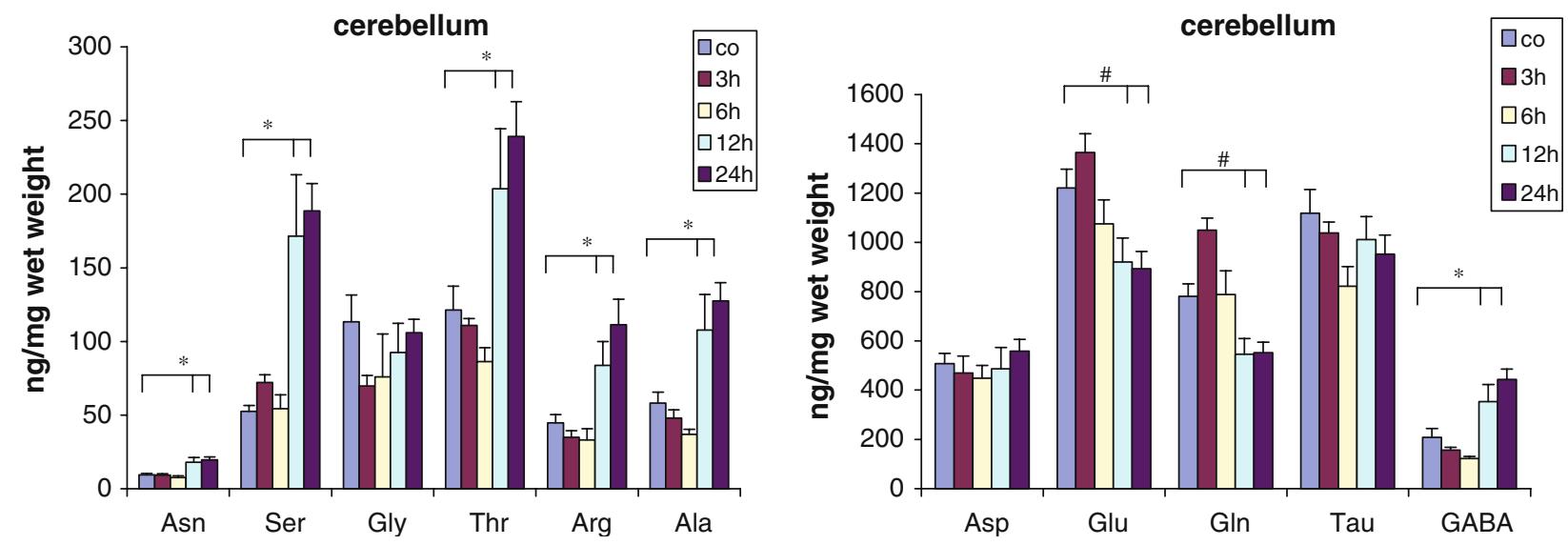

Fig. 3. Concentration of amino acids in the cerebellum after MPTP treatment ( $\mathrm{n}=7$ mice/ groups). * 12 and 24 h levels are significantly elevated compared with the controls $(P<0.05) .{ }^{\#} 12$ and $24 \mathrm{~h}$ levels are significantly decreased compared with the controls $(P<0.05$; $F(4,36)=9.5 ; 15.1 ; 0.7 ; 6.8 ; 9.9 ; 9.3 ; 0.8 ; 3.2 ; 6.3 ; 0.9 ; 8.3$ respectively). 
In the cerebellum, the Glu and Gln concentrations were decreased after 12 and $24 \mathrm{~h}$, but not after 3 and $6 \mathrm{~h}$. In contrast with these data, the levels of Asn, Ser, Thr, Arg, Ala and GABA were elevated after 12 and $24 \mathrm{~h}$. Interestingly, the Glu and Gln concentrations tended to be elevated after $3 \mathrm{~h}$, before being decreased after 12 and $24 \mathrm{~h}$, but this elevation did not reach the level of significance. The concentrations of Asp, Gly and Tau did not change significantly.

\section{After 3-NP Administration}

The level of the amino acids in different brain regions of mice treated with $3-\mathrm{NP}$ are shown in Figs. 4, 5 and 6.

The levels of Asp, Asn, Ser, Gly, Arg, Ala and GABA in the striatum were decreased after 3 and $6 \mathrm{~h}$ and these levels recovered after 12 and $24 \mathrm{~h}$. Moreover in the cases of Ser, Gly, Thr, Arg, Ala and GABA, the concentrations did not merely recover, but increased significantly as compared with the baseline levels.

The Asn, Ser, Gln, Gly, Arg, Ala and GABA levels in the cortex decreased significantly and remained low for $24 \mathrm{~h}$, although there was a tendency to a recovery after $6 \mathrm{~h}$. The Glu levels were increased after 3 and $6 \mathrm{~h}$.

In the cerebellum, the concentration of Gln was decreased after 12 and $24 \mathrm{~h}$. The Gly concentration elevated after $24 \mathrm{~h}$, while the Glu levels decreased after $12 \mathrm{~h}$. The Asn, Ser, Thr, Arg, Ala and GABA levels were elevated after 12 and $24 \mathrm{~h}$, while the Tau concentration did not alter.

\section{DISCUSSION}

The mechanism of action of mitochondrial toxins in inducing neurotoxicity involves excitotoxicity, energy deficit and free radical damage. At the concentrations we used, these toxins cause apoptotic cell death. In mice there is no detectable cell loss at $24 \mathrm{~h}$ after the toxin administration, but the apoptotic process has already started (7). Within $24 \mathrm{~h}$, many neurochemical alterations can be detected, including ATP loss, increased free radical production and the activation of apoptotic enzymes. The actual concentrations of the amino acids depend on the rates of production, elimination and transport. There are several amino acid transporters (the glutamate transporter, the neutral amino acid transporter, the excitatory amino acid transporters, the glycin transporter, the basic amino acid transporters, the cationic amino acid transporters, etc.), which require ATP for operation. There is no evidence that these mitochondrial toxins may affect these transporters including the cysteine-glutamate transporter, which is the major source of extracellular glutamate in the dosal striatum. Also the lack of ATP can effect the amino acids production and elimination. The administration of MPTP results in a rapid loss of ATP in the different brain regions. Radiolabelling studies demonstrated that the highest concentrations of radioactivity following the intravenous injection of a radiolabelled MPTP analogue were found in the caudate-putamen and the frontal, temporal and cingulate cortices; the substantia nigra and inferior olivary nucleus were labelled with medium intensity. Only very low concentrations of the radiolabelled analogue were detected in the cerebellum and white matter (12).

In the striatum the ATP levels remain low for more than $8 \mathrm{~h}$ after toxin administration (13). In contrast, the ATP levels in the cerebellar cortex return to normal within $4 \mathrm{~h}$ following MPTP exposure (14). The loss of ATP leads to an energy deficit and probably to metabolic distress. Since the synthesis of amino acids and their transport via different transporters requires energy, the ATP loss may reduce the concentrations of these amino acids. The basal amino acid levels in each brain region are similar to the previous studies. Our experiments revealed markedly decreased concentrations after MPTP administration in all the studied brain areas, including the cortex and cerebellum, which are not affected by MPTP toxicity. Both after MPTP administration and after 3-NP administration, most of the amino acid concentrations in the striatum dropped dramatically, in parallel with the ATP loss. These levels recovered after $12 \mathrm{~h}$, when the inhibition of complex I and apoptotic process start. In the cortex, which is not primarily affected by mitochondrial toxins, the concentrations remained low for $24 \mathrm{~h}$, although in the case of 3-NP the levels had started to recover. This is somewhat surprising since the ATP loss is not so pronounced and prolonged in the cortex as in the striatum. On the other hand no behavioural or motoric changes are detectable after toxin administration, despite the prolonged depression of the amino acid levels. The microdialysis studies showed no changes in the levels of Glu and 

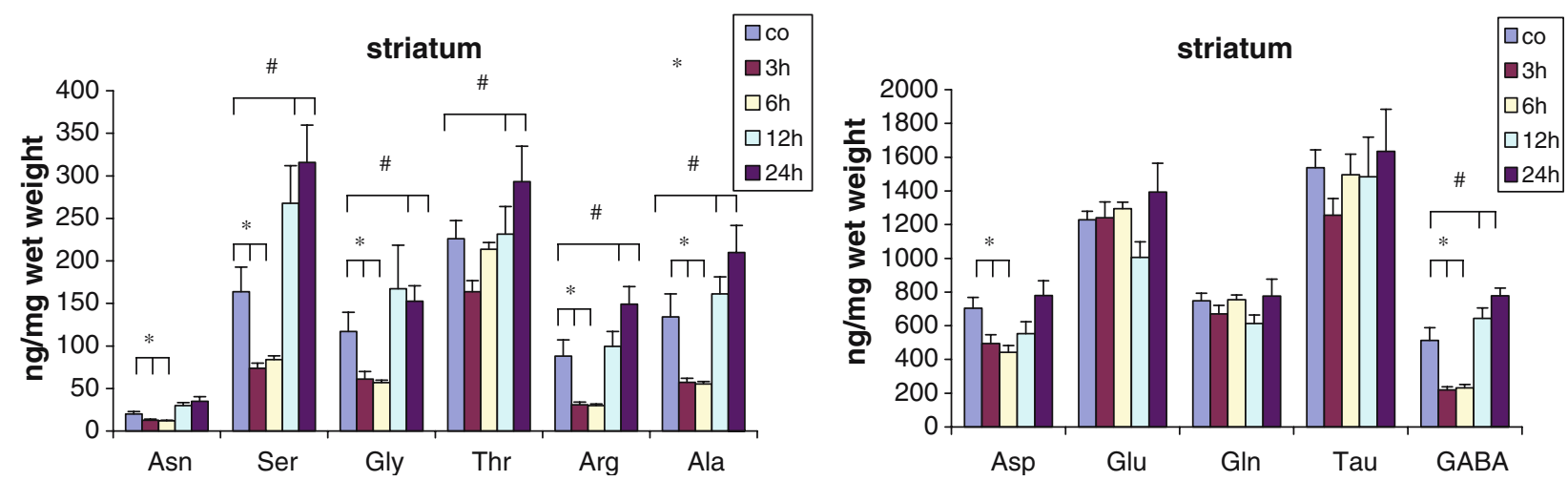

Fig. 4. Concentration of amino acids in the striatum after $3 \mathrm{NP}$ treatment $(\mathrm{n}=7$ mice/groups). $* 3$ and $6 \mathrm{~h}$ levels are significantly decreased compared with the controls $(P<0.05) .{ }^{\#} 24 \mathrm{~h}$ level is significantly increased compared with the control $(P<0.05 ; F(4,40)=7.1 ; 8.0 ; 2.6 ; 2.3 ; 5.1 ;$ $4.6 ; 3.4 ; 2.1 ; 1.1 ; 0.6 ; 8.7$ respectively).
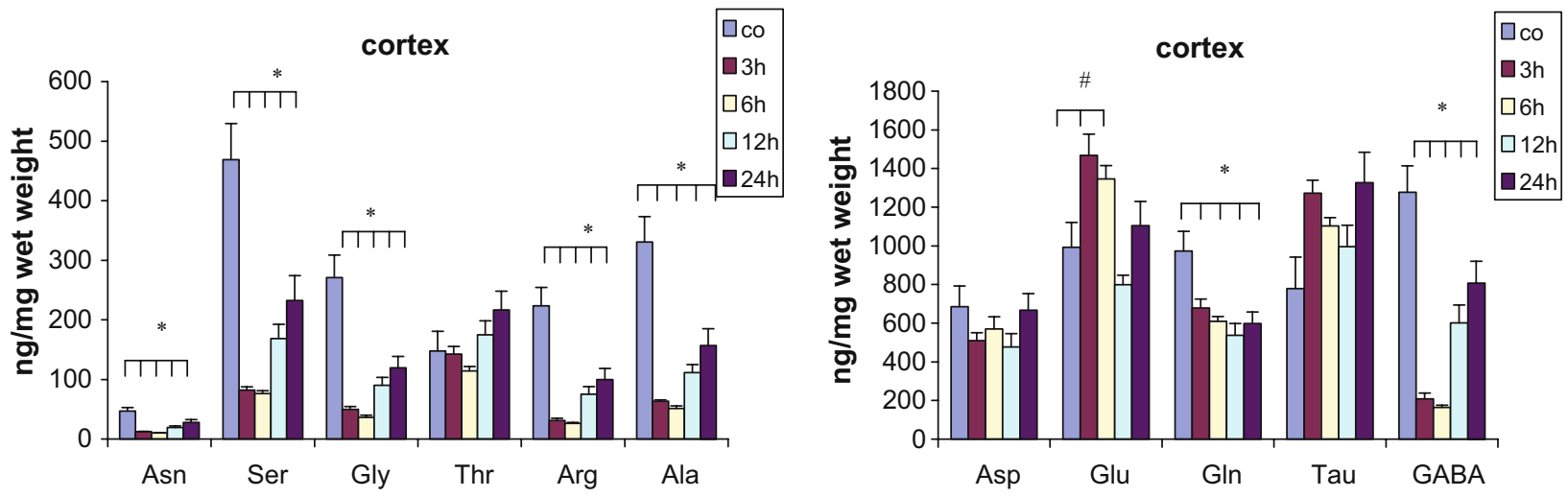

Fig. 5. Concentration of amino acids in the cortex after $3 \mathrm{NP}$ treatment ( $\mathrm{n}=7$ mice/groups). *at all time-points the levels significantly decreased compared with the controls $(P<0.05)$. ${ }^{\#} 3$ and $6 \mathrm{~h}$ levels are significantly increased compared with the control $(P<0.05$; $F(4,34)=12.8 ; 13.0 ; 10.2 ; 12.5 ; 13.1 ; 8.8 ; 5.6 ; 5.3 ; 4.7 ; 16.8$ respectively).
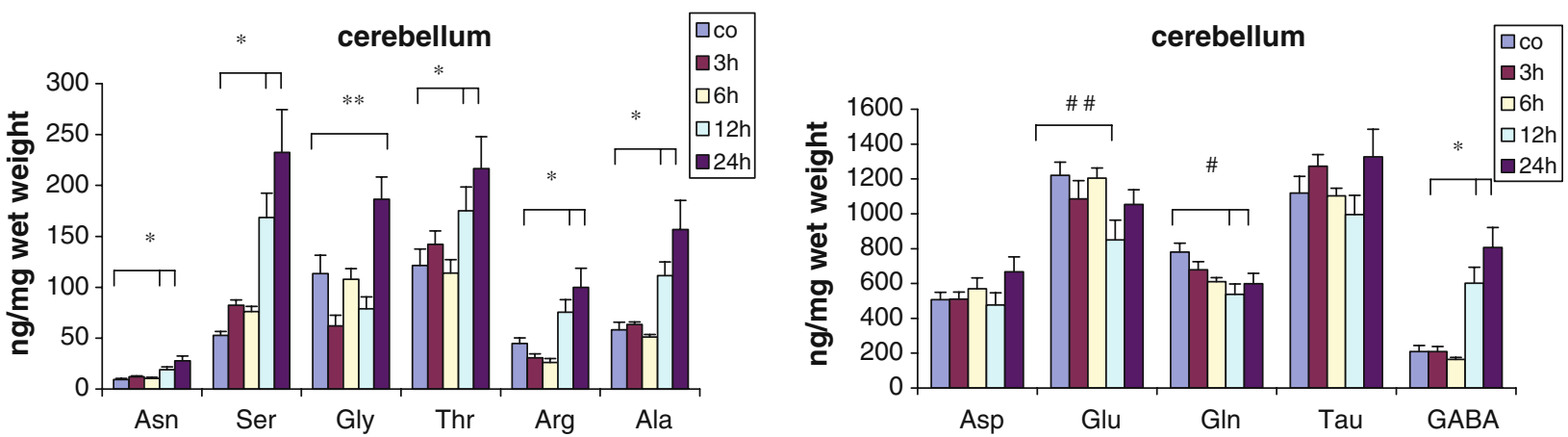

Fig. 6. Concentration of amino acids in the cerebellum after $3 \mathrm{NP}$ treatment ( $\mathrm{n}=7$ mice/groups). $* 12$ and $24 \mathrm{~h}$ levels are significantly elevated compared with the controls $(P<0.05) ;{ }^{* * 24}$ h levels are significantly increased compared with the controls $(P<0.05)$. ${ }^{\#} 12$ and 24 h levels are significantly decreased compared with the controls $(P<0.05)$. ${ }^{\#} 12 \mathrm{~h}$ levels are significantly decreased compared with the controls. $(P<0.05$ $F(4,38)=11.5 ; 18.7 ; 3.1 ; 12.1 ; 8.9 ; 14.6 ; 2.2 ; 2.2 ; 2.4 ; 1.5,9.9$ respectively $)$. 
Asp following $3 \mathrm{NP}$ administration in the striatum $(15,16)$. We did not detect changes in hte Glu levels, but found a significant reduction of Asp levels. Robinson et al. conducted a microdialysis study using MPTP (17). They found alteration in glutamate levels following MPTP administration, which we could not confirm. In these microdialysis experiments different dosage regimes and time windows were used, so the results cannot be compared.

In the cerebellum, opposite findings were detected. Following toxin administration, most of the amino acid levels were elevated after 12 and $24 \mathrm{~h}$. In the cerebellum, the ATP loss is mild and there is no evidence of mitochondrial complex inhibition, but MPTP-induced Purkinje cell toxicity is assumed (18). MPTP appears to accumulate intracellularly within the lysosomes, and, over an interval of several days, MPTP is intracellularly converted to $\mathrm{MPP}^{+}$(19). A constant, small amount of $\mathrm{MPP}^{+}$is retained intracellularly over a 72 -h interval. At low doses neither MPTP nor $\mathrm{MPP}^{+}$is neurotoxic to cultured cerebellar astrocytes, as determined by the cell counts (20). The elevations in different amino acids may reflect an overcompensation and neuroprotective mechanism via accelerated neurotransmission and neuromodulation. These findings conflict with those of the previous study by Chan et al., since they did not observe changes in Glu and Asp levels in the cerebellum. However, they used a different dose, a different mode of administration and different time points, which may explain the difference. These amino acids function as neurotransmitters and/or neuromodulators. We did not find any correlation in respect to the amino acid function and reaction to the neurotoxins.

\section{CONCLUSIONS}

In the striatum and in the cortex most of the amino acid levels (Asn, Ser, Gly, Arg, Ala, Asp, and GABA) were decreased 3 and $6 \mathrm{~h}$ after both mitochondrial toxin administration, when a marked ATP loss is detectable. So the decreased level of amino acids can be explained by the energy deficit caused by these neurotoxins. These low levels of neurotransmitters and neuromodulators may contribute to the metabolic distress and to the apoptosis. As the ATP level recovers, the amino acid concentrations start to return to the normal levels. In a later phase, as the cell loss is pronounced, certain neurotransmitter levels, such as that of dopamine, fall again (14).

\section{ACKNOWLEDGMENTS}

This work was supported by grants from the Hungarian Science Foundation (F043306), Bolyai János Research Fellowship, MediChem I. 8, AgyProt 1/4, ETT 010/2003, OM Bio-00100/2002 and RET-NORT 08/2004. We thank David Durham from England for his linguistic corrections.

\section{REFERENCES}

1. Beal, M. F. 1994. Huntington's disease, energy, and excitotoxicity. Neurobiol. Aging 15:275-276.

2. Rego, A. C. and Oliveira, C. R. 2003. Mitochondrial dysfunction and reactive oxygen species in excitotoxicity and apoptosis: implications for the pathogenesis of neurodegenerative diseases. Neurochem. Res. 28:1563-1574.

3. Gardian, G. and Vecsei, L. 2004. Huntington's disease: pathomechanism and therapeutic perspectives. J. Neural. Transm. 111:1485-1494.

4. Beal, M. F. 2000. Energetics in the pathogenesis of neurodegenerative diseases. Trends Neurosci. 23:298-304.

5. Klivenyi, P., Andreassen, O. A., Ferrante, R. J., Lancelot, E., Reif, D., and Beal, M. F. 2000. Inhibition of neuronal nitric oxide synthase protects against MPTP toxicity. Neuroreport $11: 1265-1268$

6. Klivenyi, P., Starkov, A. A., Calingasan, N. Y., Gardian, G., Browne, S. E., Yang, L., Bubber, P., Gibson, G. E., Patel, M. S., and Beal, M. F. 2004. Mice deficient in dihydrolipoamide dehydrogenase show increased vulnerability to MPTP, malonate and 3-nitropropionic acid neurotoxicity. J. Neurochem. 88:1352-1360.

7. Przedborski, S., Tieu, K., Perier, C., and Vila, M. 2004. MPTP as a mitochondrial neurotoxic model of Parkinson's disease. J. Bioenerg. Biomembr. 36:375-379.

8. Brouillet, E., Conde, F., Beal, M. F., and Hantraye, P. 1999. Replicating Huntington's disease phenotype in experimental animals. Prog. Neurobiol. 59:427-468.

9. Marcaggi, P. and Attwell, D. 2004. Role of glial amino acid transporters in synaptic transmission and brain energetics. Glia 47:217-225.

10. Chan, P., Di Monte, D. A., and Langston, J. W. 1994. Effects of 1-methyl-4-phenyl-1,2,3,6-tetrahydropyridine (MPTP) on levels of glutamate and aspartate in the mouse brain. Brain Res. 647:249-254

11. Klivenyi, P., Kekesi, K., Juhasz, G., and Vecsei, L. 1997. Amino acid concentrations in cerebrospinal fluid of patients with multiple sclerosis. Acta Neurol. Scand. 95:96-98.

12. Efange, S. M., Kung, H. F., Mash, D. C., Jabir, M., Billings, J., Pablo, J., Dutta, A., and Freshler, A. 1990. Pargyline-sensitive selective accumulation of a radiolabeled MPTP analog in the primate cerebral cortex and basal ganglia. Synapse 5:207-212.

13. Chan, P., Langston, J. W., Irwin, I., DeLanney, L. E., and Di Monte, D. A. 1993. 2-deoxyglucose enhances 1-methyl-4 phenyl-1,2,3,6-tetrahydropyridine-induced ATP loss in the mouse brain. J. Neurochem. 61:610-616.

14. Di Monte, D. A., Wu, E. Y., and Langston, J. W. 1992. Role of astrocytes in MPTP metabolism and toxicity. Ann. N.Y. Acad. Sci. 648:219-228. 
15. Beal, M. F., Brouillet, E., Jenkins, B. G., Ferrante, R. J., Kowall, N. W., Miller, J. M., Storey, E., Srivastava, R., Rosen, B. R., and Hyman, B.T. 1993. Neurochemical and histologic characterization of striatal excitotoxic lesions produced by the mitochondrial toxin 3-nitropropionic acid. J. Neurosci. 13:4181-1492.

16. Sanchez-Carbente, M. R. and Massieu, L. 1999. Transient inhibition of glutamate uptake in vivo induces neurodegeneration when energy metabolism is impaired. J. Neurochem. 72:129-138.

17. Robinson, S., Freeman, P., Moore, C., Touchon, J. C., Krentz, L., and Meshul, C. K. 2003. Acute and subchronic MPTP administration differentially affects striatal glutamate synaptic function. Exp. Neurol. 180:74-87.

18. Takada, M., Sugimoto, T., and Hattori, T. 1993. MPTP neurotoxicity to cerebellar Purkinje cells in mice. Neurosci. Lett. 150:49-52.

19. Marini, A. M, Lipsky, R. H., Schwartz, J. P., and Kopin, I. J. 1992. Accumulation of 1-methyl-4-phenyl-1,2,3,6-tetrahydropyridine in cultured cerebellar astrocytes. J. Neurochem. 58:1250-1258.

20. Marini, A. M., Lipsky, R. H., Schwartz, J. P., and Kopin, I. J. 1992. Accumulation of 1-methyl-4-phenyl-1,2,3,6-tetrahydropyridine in cultured cerebellar astrocytes. J. Neurochem. 58:1250-1258. 